\title{
Characteristics of the Thermal Strain of Gas Jet-Assisted Laser Percussion Drilling
}

\author{
Chao-Ching Ho, ${ }^{*}$ Yuan-Jen Chang, ${ }^{1}$ Jin-Chen Hsu, ${ }^{1}$ Chia-Lung Kuo, ${ }^{1}$ and Guan-Hong Lee ${ }^{1}$ \\ Graduate Institute of Manufacturing Technology and Department of Mechanical Engineering, \\ National Taipei University of Technology, 1, Sec. 3, Zhongxiao E. Rd., Taipei 10608, Taiwan, R.O.C. \\ ${ }^{1}$ Department of Mechanical Engineering, National Yunlin University of Science and Technology, \\ 123 University Road, Section 3, Douliou, Yunlin 64002, Taiwan, R.O.C.
}

(Received August 15, 2015; accepted June 14, 2016)

Keywords: hole drilling, residual thermal strain measurement, digital image correlation, ring-core method

In this work, a digital image correlation technique in combination with laser hole drilling to measure the residual thermal strain is presented. The significant potential benefits of this work are non-contact, high speed, and on-line measurement. The ring-core based groove in the surface of a specimen was drilled concentrically to induce thermal stresses inside the core. These deformations can be measured by a digital image correlation technique for measuring relieved strains. We also investigated the size of the reference areas on the specimens and tracked these areas after the laser hole drilling process. The proposed method was applied to characterize the thermal effect produced by laser milling for aluminum alloy A15052 under plane strain tension conditions, and the results were compared with four assisted laser milling approaches.

\section{Introduction}

Digital image correlation (DIC) is a non-contact optical technique used to measure displacements on the surface of an object by performing correlation analysis of random patterns on surfaces created by spraying with an airbrush gun. A critera for quantitative evaluation of random patterns and the characterization of effectiveness of DIC algorithm implementation and pattern printing on the surfaces of interest are introduced.(1) A practical approach for use in a mechanical testing laboratory to optimize the performance of DIC was proposed. ${ }^{(2)}$ This approach allowed estimation of the average speckle size, as well as the scatter of speckle sizes, to maximize spatial resolution. Two-dimensional (2D) DIC provides full-field displacements to sub-pixel accuracy and full-field strains by comparing the digital images of a test object surface acquired before and after deformation. ${ }^{(3)}$ Therefore, stress can be identified based on the application of the 2D DIC technique. The analysis of the stress release is achieved by DIC applied to load state SEM images captured in a combination of scanning electron microscopy (SEM) and focused ion beam (FIB) equipment. ${ }^{(4)}$ The FIB micro-hole milling was combined with 2D finite-element analysis (FEA) to model the resulting relaxation displacements on the specimen surface based on the DIC analysis. ${ }^{(5)}$ The measurement of the displacement field based on the digital image correlation originating when a slot is milled into the material was presented.( ${ }^{(6)}$ DIC for determining the coefficient of thermal expansion (CTE) of films was investigated. ${ }^{(7)}$ Hence, DIC has been established as a practical and "Corresponding author: e-mail: HoChao@mail.ntut.edu.tw 
effective deformation measurement technique. To the authors' knowledge, measurement of the residual thermal strain using a DIC method has not been previously reported. The measurement of thermal stress is important because it alters mechanical and functional performance. Thermal stress and strain fields are developed during laser milling processes. 2D DIC can be used directly to quantitatively determine the deformation field thermal strain. The ring-core method is a partially destructive test method for measuring uniform and non-uniform residual stresses. The major advantages of the ring-core method is its greater sensitivity. For residual stress measurements, the ring-core method using the strain gauge enables the profile of residual stresses in materials to be determined up to a depth of $5 \mathrm{~mm} .{ }^{(8)}$ However, a strain gauge uses a pointwise technique; but the drawback of applying a strain gage rosette is that the machining axis must be aligned with the center of the strain gage rosette. The aim of this study was to use different assisted laser-holedrilling mechanisms based on the ring-core method to characterize the induced residual thermal strain by DIC.

\section{DIC Method and Hole-Drilling}

In the DIC method, two speckle images are compared. The initial (reference) image with the calculation area (region of interest, i.e., ROI) is divided into evenly spaced subsets. The ROI is the reference region in which the displacement/deformation field is determined. The subset is a square matrix of pixels used as the basis of the image correlation technique. Matching each initial subset to the respective calculated subset in the deformed image is carried out by defining the maximum value of the correlation coefficient. The image is transformed into grey scale. Specimens are cut from an aluminum sheet (i.e., Al 5052) $6 \mathrm{~mm}$ thick, and the area of the specimens is $20 \times 70$ $\mathrm{mm}^{2}$. A digital camera JAI BM-500GE (5 megapixel matrix) and a camera lens (focal length 105 $\mathrm{mm}$, aperture $\mathrm{f} / 2.8-32$ ) are used to register the working surface of the patterned specimens during testing. The camera's optical axis is adjusted to be orthogonal to the studied surfaces, which are illuminated with red light. The area of the pattern captured was approximately $20 \times 16 \mathrm{~mm}^{2}$. Digital image correlation was performed on a selected $(160 \times 160$ pixels $)$ sub-image of the surface captured by a digital camera with $122 \mathrm{pixel} / \mathrm{mm}$ resolution. Figures 1 (a) and 1 (b) show a schematic illustration of the experimental setup and the actual experimental system using an optical imaging

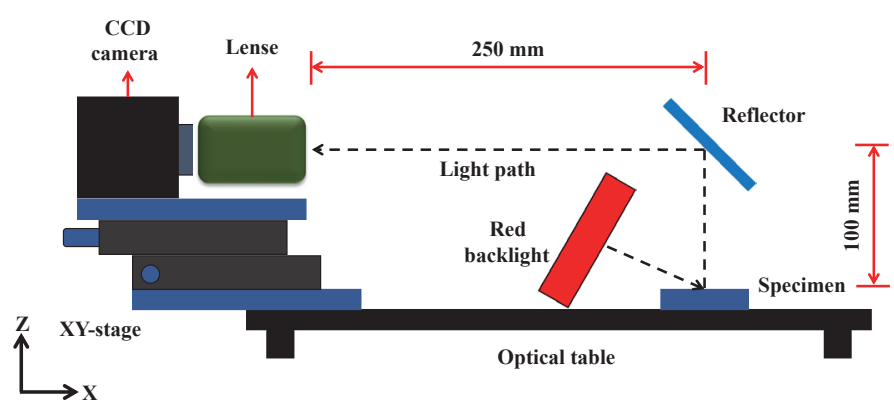

(a)

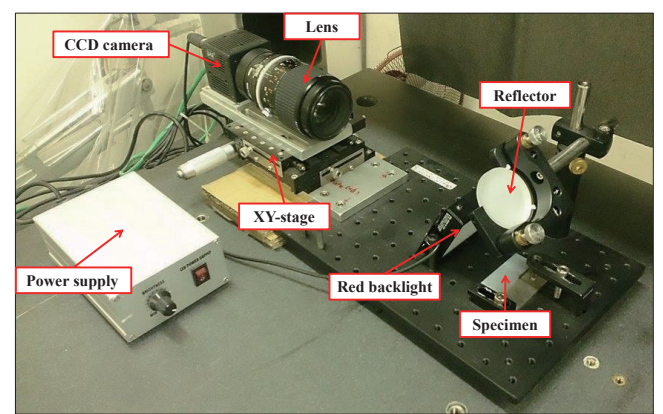

(b)

Fig. 1. (Color online) (a) Schematic illustration of the experimental setup and (b) actual experimental setup. 
device for the 2D DIC method, respectively. These images were analyzed by the DIC technique to extract the induced strains after milling. The arrangement used for studying the effect of the induced residual thermal strain is shown in Fig. 2 and Table 1. The entire experimental system used was established on a micro-precision electrical discharge machining (EDM) machine (Sodick AP1L). A fixture connected the holders of the optical components (a mirror and a lens) to the column of the EDM machine. The mirror and focal lens were positioned in each precision stage to tune the laser direction and adjust the focal point of the laser. The mirror reflected the laser to the sample while a lens with a z-axis stage focused the laser on the workpiece. A lens with a focal length of $120 \mathrm{~mm}$ was used for the focusing. The laser beam was focused negatively (i.e., -0.3 $\mathrm{mm}$ ) below the material's surface, providing a beam spot approximately $12.9 \mu \mathrm{m}$ in diameter. The laser source was a Nd:YAG laser (LOTIS LS-2134UTF) providing a Gaussian laser beam and four harmonic modes, i.e., four different wavelengths. The maximum beam diameter of the output was $6 \mathrm{~mm}$, and the beam divergence was typically below $0.8 \mathrm{mrad}$. A 532-nm wavelength with pulse mode was used in all experiments. The maximum energy of a single pulse was $200 \mathrm{~mJ}$, and the maximum frequency was $15 \mathrm{~Hz}$. The pulse width was approximately $6 \mathrm{~ns}$ for Nd:YAG lasers.

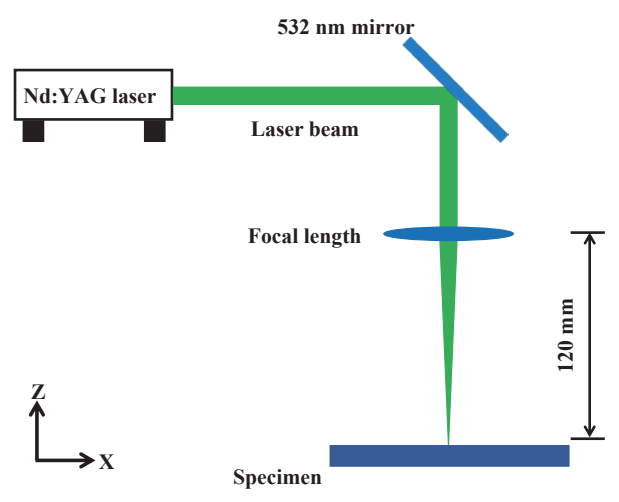

(a)

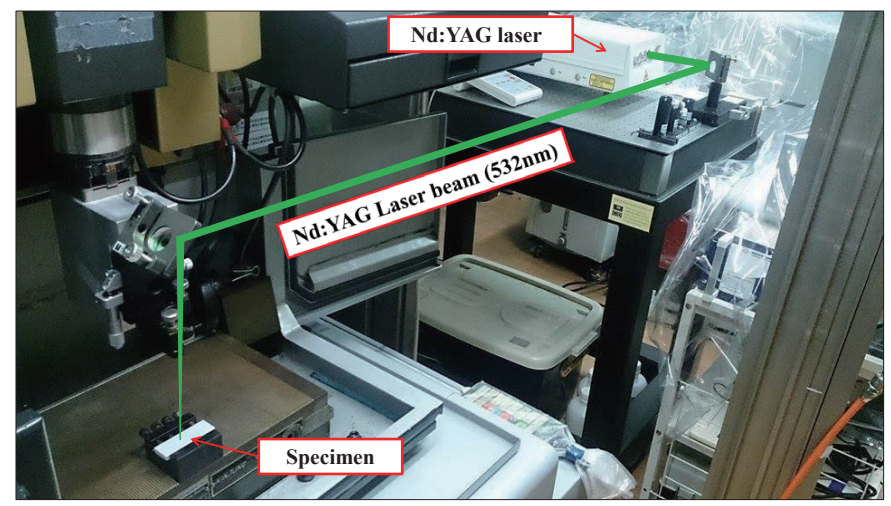

(b)

Fig. 2. (Color online) (a) Schematic illustration of the laser milling setup and (b) actual experimental setup.

Table 1

Laser machining parameters.

\begin{tabular}{lc}
\hline Item & Parameter \\
\hline Wavelength & $532 \mathrm{~nm}$ \\
Frequency & $15 \mathrm{~Hz}$ \\
Pumping lamp energy & $21 \mathrm{~J}$ \\
Radiation energy & $200 \mathrm{~mJ}$ \\
Focal length & $120 \mathrm{~mm}$ \\
Milling speed & $2 \mathrm{~mm} / \mathrm{min}$ \\
Processing time & $40 \mathrm{~min}$ \\
\hline
\end{tabular}




\section{Experiments and Discussion}

The laser milling of a surface slot on a specimen induces a local thermal strain release that generates a displacement field in the direction perpendicular to the slot plane. The implementation of the 2D DIC method consists of the following three consecutive steps:

(1) Specimen and experimental preparation for the reference image: The speckle pattern sprayed by an airbrush was made on the test specimen surface as a carrier of deformation information.

Figure 3 shows the speckle image of the specimen surface after being painted with white and black paint. During the DIC analysis of the captured images, a square area with a size of $160 \times$ 160 pixels in the middle of the specimen was chosen to be the region of interest.

(2) Recording images of the planar surface of the specimen before and after machining: in the first step, a DIC image of the reference area to be analyzed was captured by the camera. The dimensions of the scanned area were determined. After capturing the DIC reference image, different assisted laser milling methods were used to induce residual thermal strain by milling a slot on the region of interest. The DIC image of the area around the milled zone was then captured and analyzed.

(3) Processing the acquired images using a DIC program to obtain the desired displacement and strain information: The DIC program compared grey level intensity patterns of a matrix of pixels of both images at sub-pixel locations and established where the reference matrix has to be shifted, from the first to the second image, to find the best matching pattern.

Figure 4 shows photographs of the different assisted laser milling equipment. Four different assisted configurations - no gas jet, swirling gas jet $\left(25^{\circ} \mathrm{C}\right),{ }^{(9)}$ swirling gas jet $\left(0^{\circ} \mathrm{C}\right)$, and straight gas jet $\left(0^{\circ} \mathrm{C}\right)^{(10)}$ for enhancing thermal efficiency-were investigated for their effect on the thermal strain of percussion drilling of $6 \mathrm{~mm}$ thick aluminum sheets. Strain range is defined as the difference between maximum and minimum displacement values between the reference and the deformed images after DIC calculation. Figures 5-8 show the displacement field in the $x$ and $y$ directions obtained by the correlation of two images under four different assisted configurations.

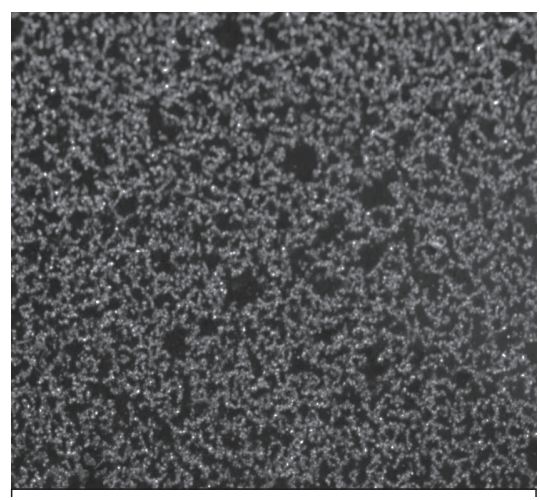

(a)

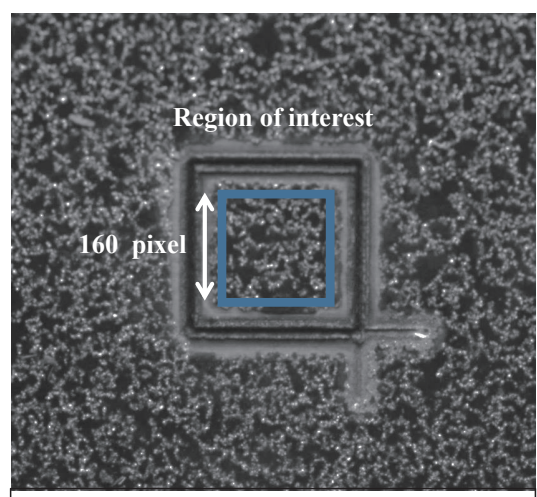

(b)

Fig. 3. (Color online) Surface speckle images of the specimen (a) before and (b) after machining. The area inside the blue rectangle is the calculation area. 


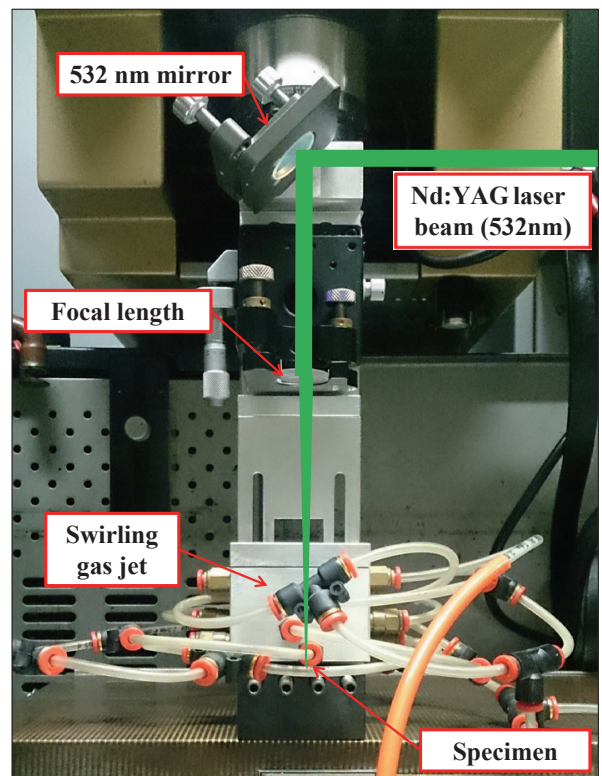

(a)

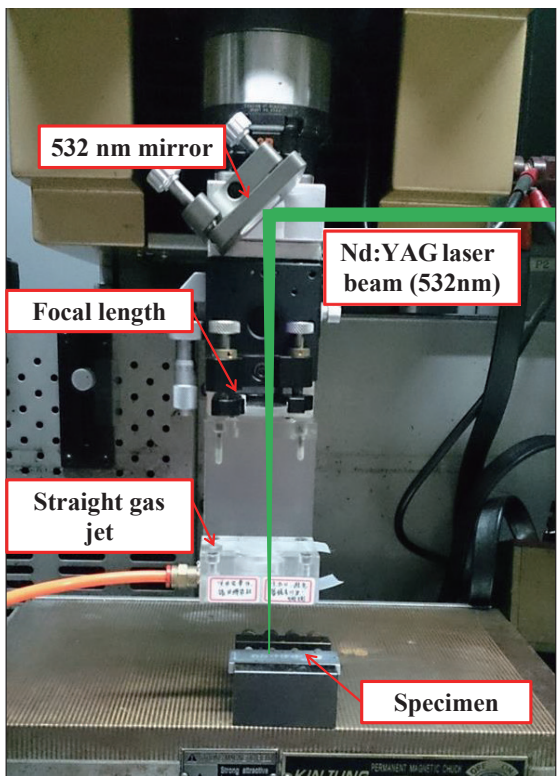

(b)

Fig. 4. (Color online) Experimental setup: (a) swirling gas jet and (b) straight gas jet for the residual thermal strain observation for different assisted laser percussion drilling configurations.
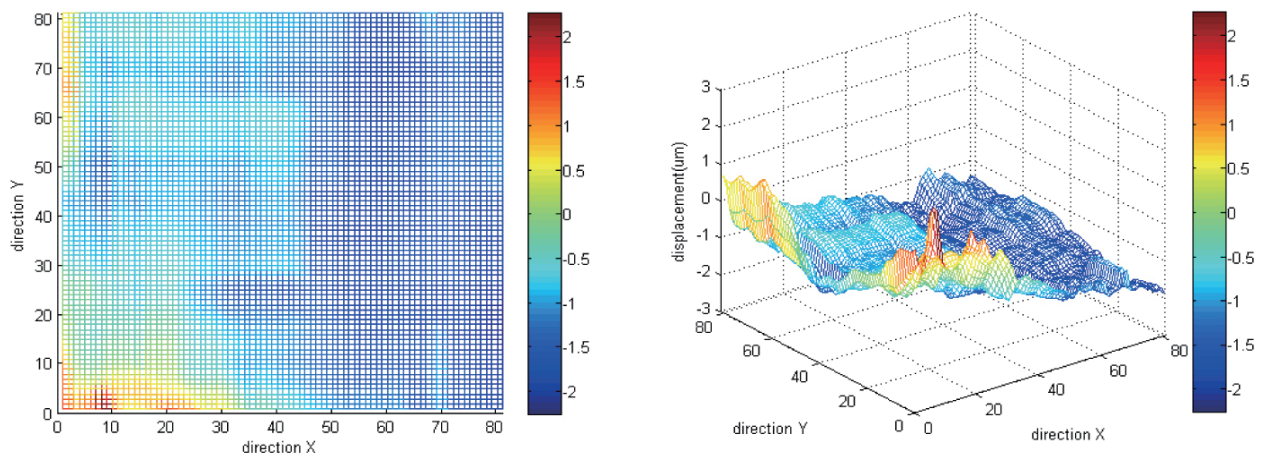

(a)
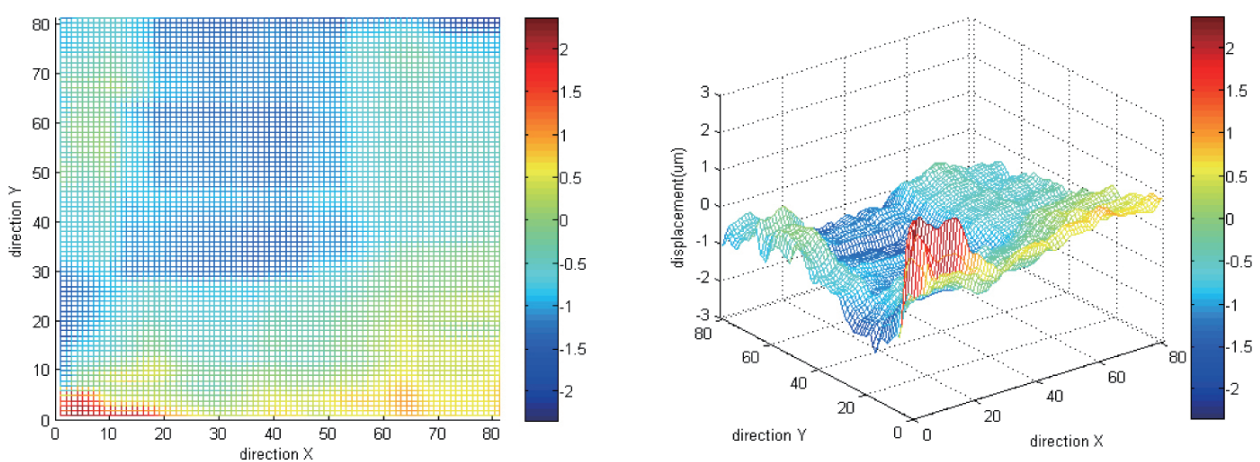

(b)

Fig. 5. (Color online) The displacement field in the $x$ and $y$ directions for the no-gas jet configuration. (a) The displacement field in the $x$ direction and strain range of $4.53 \mu \mathrm{m}$. (b) The displacement field in the $y$ direction and strain range of $4.70 \mu \mathrm{m}$. 

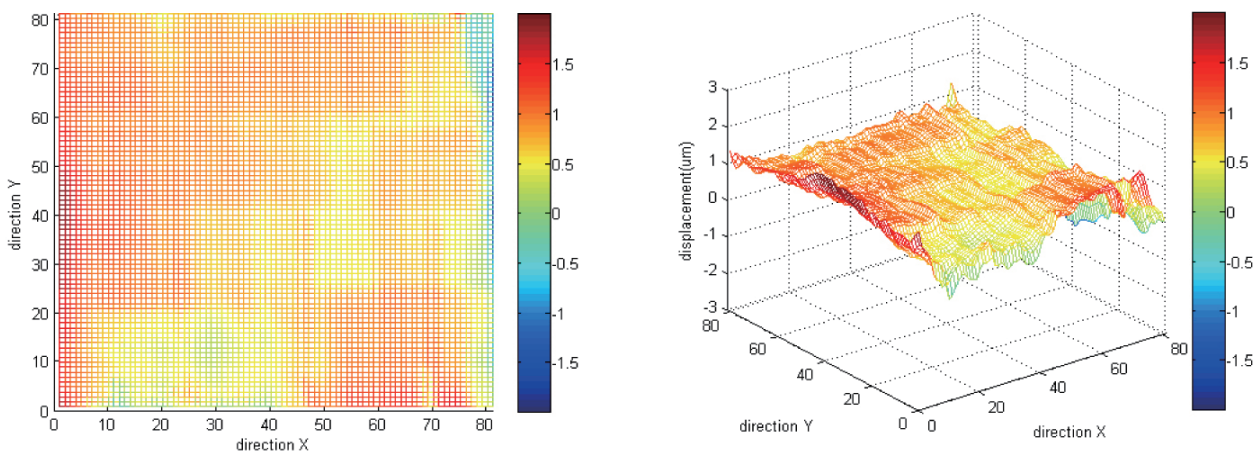

(a)
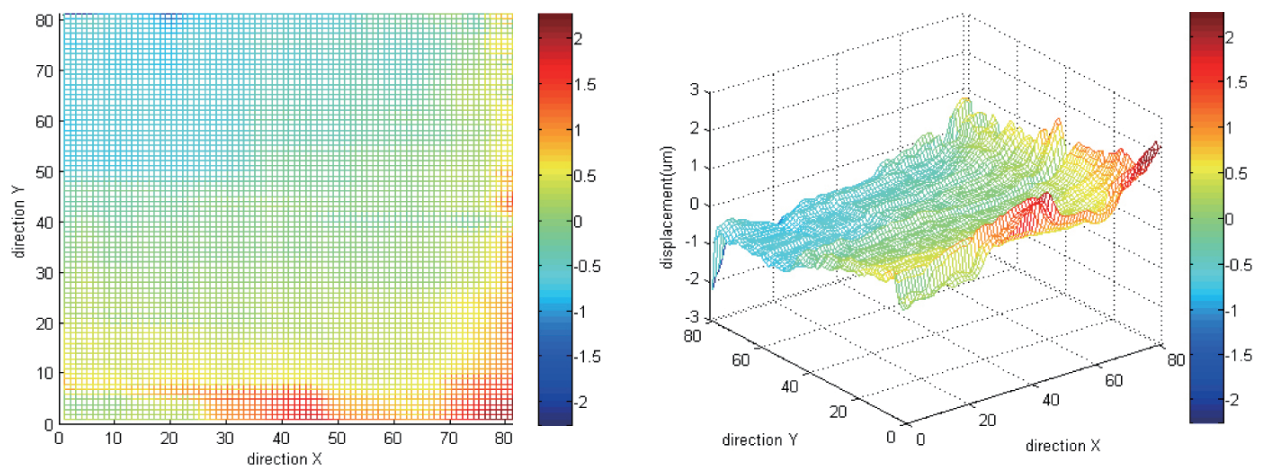

(b)

Fig. 6. (Color online) The displacement field in the $x$ and $y$ directions for the swirling gas jet $\left(25^{\circ} \mathrm{C}\right)$ configuration. (a) The displacement field in the $x$ direction and strain range of $3.99 \mu \mathrm{m}$. (b) The displacement field in the $y$ direction and strain range of $4.50 \mu \mathrm{m}$.
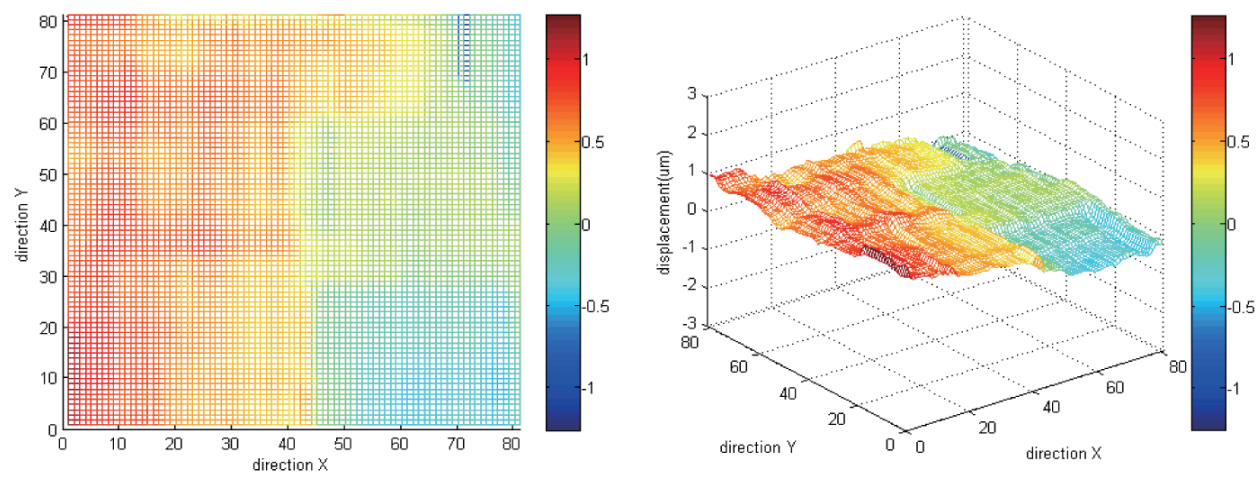

(a)
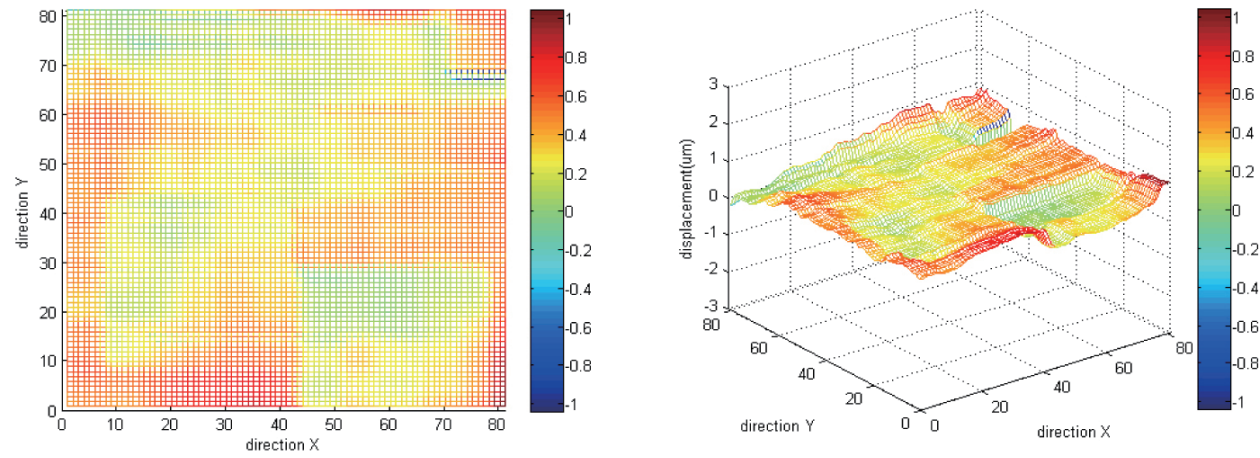

(b)

Fig. 7. (Color online) The displacement field in the $x$ and $y$ directions for the swirling gas jet $\left(0^{\circ} \mathrm{C}\right)$ configuration. (a) The displacement field in the $x$ direction and strain range of $2.51 \mu \mathrm{m}$. (b) The displacement field in the $y$ direction and strain range of $2.08 \mu \mathrm{m}$. 

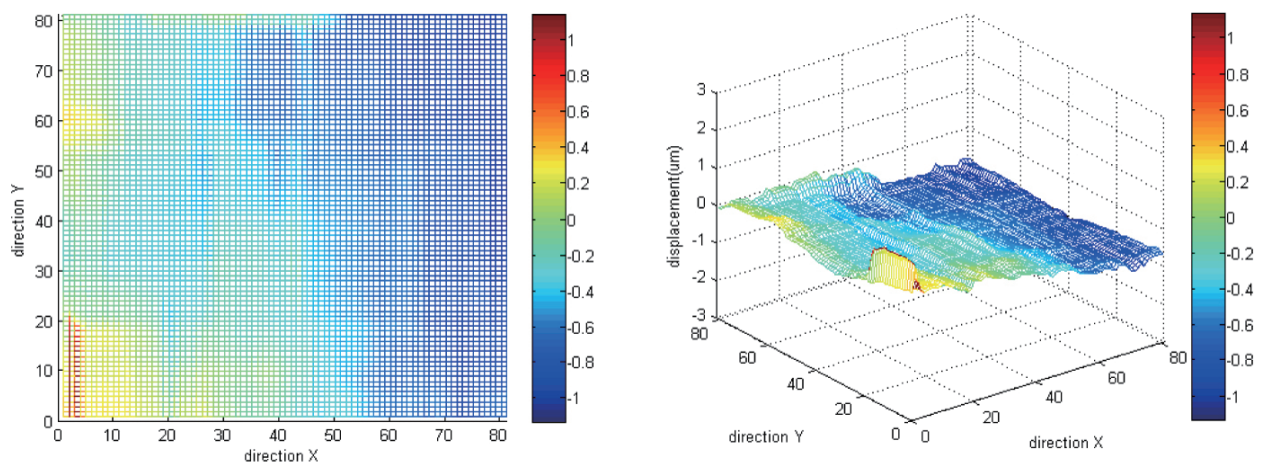

(a)
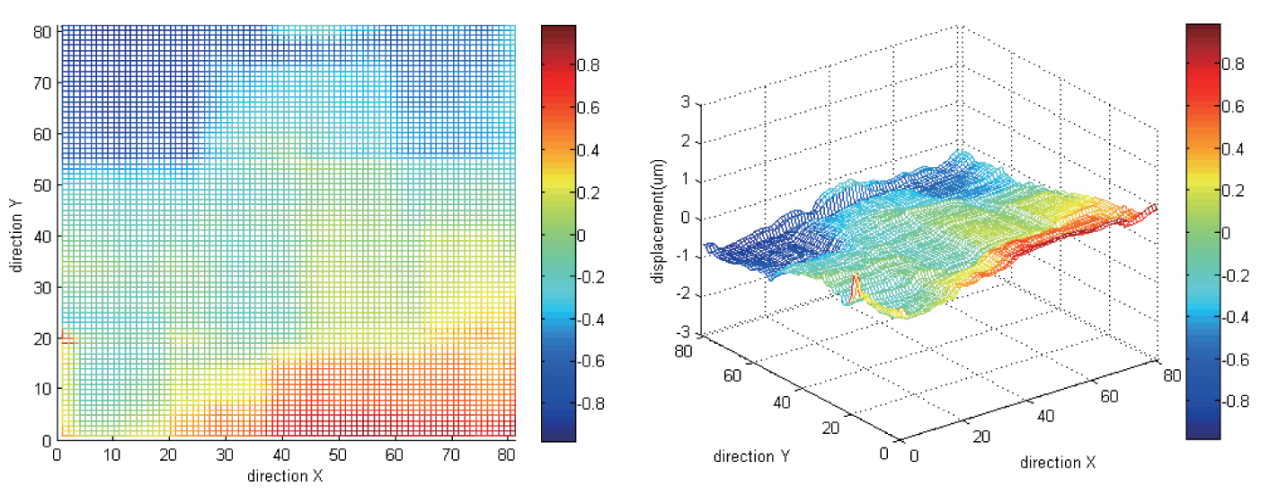

(b)

Fig. 8. (Color online) The displacement field in the $x$ and $y$ directions for the straight gas jet $\left(0^{\circ} \mathrm{C}\right)$ configuration. (a) The displacement field in the $x$ direction and strain range of $2.27 \mu \mathrm{m}$. (b) The displacement field in the $y$ direction and strain range of $1.97 \mu \mathrm{m}$.

The laser induced thermal strain was smallest for the swirling gas jet $\left(0{ }^{\circ} \mathrm{C}\right)$ and straight gas jet $\left(0{ }^{\circ} \mathrm{C}\right)$ as the thermal energy produced was conducted efficiently. The swirling gas jet $\left(25^{\circ} \mathrm{C}\right)$ produced less thermal strain than the no-gas jet assisted configuration because the assisted gas could remove much more thermal energy. The no-gas jet assisted configuration produced the most strain, as expected, due to the thermal effect of the laser.

\section{Conclusions}

This work presents a new method for mapping in-plane applied residual thermal strain incrementally as a function of depth based on hole drilling. Full-field in-plane deformation fields associated with the machining change were measured by the DIC technique. Al 5052 was employed as a reference sample to verify the feasibility and effectiveness of the proposed method. The swirling gas jet $\left(0^{\circ} \mathrm{C}\right)$ and straight gas jet $\left(0^{\circ} \mathrm{C}\right)$ assisted laser drilling can remove the most thermal energy; the swirling gas jet $\left(25^{\circ} \mathrm{C}\right)$ outperformed the no-gas jet assisted laser drilling because the latter produced the most thermal strain produced, as expected. 


\section{Acknowledgements}

This work was supported by the Ministry of Science and Technology, Taiwan, R.O.C., MOST 103-2221-E-027-135-MY2.

\section{References}

1 G. Stoilov, V. Kavardzhikov, and D. Pashkouleva: J. Theor. Appl. Mech. 42 (2012) 55.

2 G. Lionello and L. Cristofolini: Meas. Sci. Technol. 25 (2014) 107001.

3 B. Pan, K. Qian, H. Xie, and A. Asundi: Meas. Sci. Technol. 20 (2009) 062001.

4 J. Keller, A. Gollhardt, D. Vogel, E. Auerswald, N. Sabate, J. Auersperg, and B. Michel: Mater. Sci. Forum 524 (2006) 121.

5 B. Winiarski and P. Withers: Exp. Mech. 52 (2012) 417.

6 N. Sabaté1, D. Vogel, A. Gollhardt, J. Marcos, I. Gràcia, C. Cané, and B. Michel: Nanotechnology 17 (2006) 5264.

7 B. Pan, H. M. Xie, H. Tao, and A. Asundi: Polym. Test. 28 (2009) 75.

8 E. Valentini, A. Benincasa, and L. Bertelli: Proc. 40th National Conference of the Italian Association for Stress Analysis 2011 (Italian Stress Analysis Association, 2011) pp. 1-11.

9 C.-C. Ho, Y.-M. Chen, J.-C. Hsu, Y.-J. Chang, and C.-L. Kuo: J. Laser Appl. 27 (2015) 042001.

10 J.-C. Hsu, W.-Y. Lin, Y.-J. Chang, C.-C. Ho, and C.-L. Kuo: Int. J. Adv. Manuf. Tech. 79 (2015) 449.

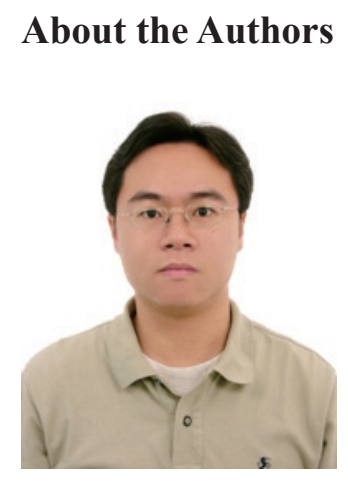

Chao-Ching Ho received his B.S. and M.S. degrees from National Taiwan University, Taipei, Taiwan, R.O.C., in 1995 and 1997, respectively, and his $\mathrm{Ph} . \mathrm{D}$. degree in electrical engineering from the National Taiwan University of Science and Technology, Taipei, Taiwan, 2008. From 2006 to 2009 , he was a senior designer of home entertainment department at STMicroelectronics. From 2009 to 2016, he was with the Department of Mechanical Engineering, the National Yunlin University of Science and Technology, where he was an associate professor. Since 2016, he has been with the Graduate Institute of Manufacturing Technology and Department of Mechanical Engineering, the National Taipei University of Technology, where he is currently an associate professor. His research interests are metrology, prognostic systems and laser ablation, as well as machine vision. He is a member of the Society of Manufacturing Engineers (SME) in Taipei.

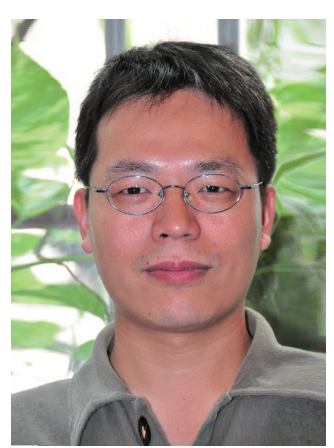

Yuan-Jen Chang received his B.S. and M.S. degrees from National Taiwan University and National Cheng Kung University, Taiwan, in 1996 and 1998, respectively. He received his Ph.D. degree from the University of Colorado at Boulder in 2008. From 2009 to 2013, he was an assisting professor of the Department of Mechanical Engineering, the National Yunlin University of Science and Technology, where he is currently an associate professor. His research interests are nanoimprint, nanofabrication technologies, and laser material processing. 


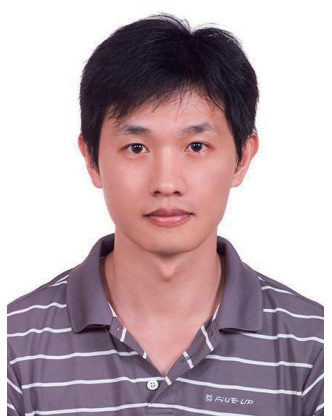

Jin-Chen Hsu received his B.S. degree in mechanical engineering from the National Taiwan University of Science and Technology in 1997 and his Ph.D. degree in applied mechanics from the National Taiwan University in 2007. From 2007 to 2009, he was a postdoctoral research fellow of the Institute of Applied Mechanics of National Taiwan University. He joined the faculty of the Department of Mechanical Engineering, the National Yunlin University of Science and Technology, Taiwan, and he is currently an associate professor. He is also a member of the Society of Theoretical and Applied Mechanics of the Republic of China. His research interests include acoustic waves in piezoelectric media and metamaterials, finite-element simulations, and laser machining.

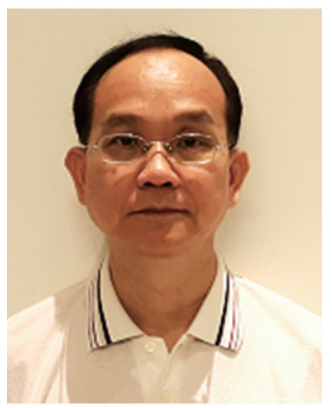

Chia-Lung Kuo received his B.S. degree from National Chung Hsing University, Taichung, Taiwan, R.O.C., in 1984 and his M.S. and Ph.D. degrees from the University of Tokyo, Japan, in 1989 and 1992, respectively. From 1992 to 2010, he was an associate professor at the Department of Mechanical Engineering, the National Yunlin University of Science and Technology, Taiwan. Since 2010, he has been a professor at the same University. His research interests are EDM, ECM, and laser processing.

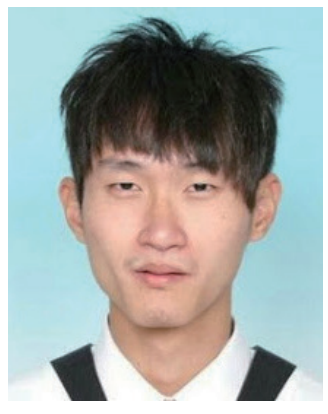

Guan-Hong Lee received his B.S. degree from National Dong Hwa University, Hualien, Taiwan, R.O.C., in 2014, and his M.S. degree in mechanical engineering from the National Yunlin University of Science and Technology, Yunlin, Taiwan, 2016. His research interests are laser ablation and machine vision. 